\title{
Diagnóstico da qualidade analítica na quantificação de cianobactérias
}

\section{Diagnostic of analytical quality in quantification of cyanobacteria}

\author{
Carla Cristine Müller \\ Mestre em Ecologia pela Universidade Federal do Rio Grande do Sul (UFRGS). Bióloga da Companhia Riograndense de Saneamento (CORSAN) \\ Maria Teresa Raya-Rodriguez \\ Química. Doutora em Ecologia e Recursos Naturais pela Universidade Federal de São Carlos (UFScar). Professora associada da UFRGS

\section{Luiz Fernando Cybis} \\ Doutor em Engenharia Sanitária e Ambiental pela University of Leeds, United Kingdom. Professor, orientador, pesquisador e consultor do Instituto de \\ Pesquisas Hidráulicas (IPH) da UFRGS
}

\section{Resumo}

O aumento de florações de cianobactérias em mananciais utilizados para abastecimento é uma preocupação global. Seguindo as diretrizes da Organização Mundial da Saúde, a portaria n 518/2004 do Ministério da Saúde, determina o monitoramento de cianobactérias nos mananciais superficiais e, quando for o caso, na água tratada. No Brasil, não há padronização de métodos de análise; assim, os métodos utilizados devem fornecer resultados confiáveis. Por meio da participação voluntária, um programa de ensaios de proficiência em cianobactérias foi realizado em 2010. Este trabalho apresenta o diagnóstico do desempenho dos laboratórios na identificação e contagem de cianobactérias. Os resultados das contagens foram submetidos ao cálculo do escore z, obtendo-se $80 \%$ de laboratórios com desempenho satisfatório. Divergências foram observadas na identificação dos gêneros de cianobactérias.

Palavras-chave: interlaboratorial; cianobactérias; qualidade analítica; análise quantitativa.

\begin{abstract}
The increase of cyanobacterial blooms in water sources used for supply is a global concern. Following the guidelines of the World Health Organization, ordinance $n^{\circ} 518 / 2004$, the of the Ministry of Health, provides the monitoring of cyanobacteria in superficial water sources and, when appropriate, in treated water. In Brazil, there is no standardization of analysis methods; thus, the applied methods should provide reliable results. Through voluntary participation, a proficiency testing program in cyanobacteria was conducted in 2010. This paper presents the diagnostic performance of laboratories in identification and counting of cyanobacteria. Results of counts were subjected to the calculation of z-score, and $80 \%$ of laboratories presented satisfactory performance. Differences were observed in the identification of cyanobacterial genera.
\end{abstract}

Keywords: interlaboratory; cyanobacteria; analytical quality; quantitative analysis

\section{Introdução}

A quantificação do fitoplâncton, em termos de células. $\mathrm{mL}^{-1}$ e/ou biomassa e a sua composição taxonômica são parâmetros importantes para avaliar a qualidade da água de mananciais superficiais. A abundância e a composição da comunidade fitoplanctônica fornecem informações sobre a saúde dos ecossistemas, bem como o risco à saúde humana. Isso por que alguns organismos (especialmente cianobactérias) produzem uma ampla variedade de compostos alergênicos, tóxicos e carcinogênicos (GREGOR et al., 2005). Em função disso, a qualidade das águas superficiais ao redor do mundo tem recebido atenção redobrada, pois a ausência de políticas e regulamentações para o uso da água faz com que sua degradação (antrópica, bioquímica ou por organismos vivos) inviabilize o uso a longo prazo (LEBOULANGER et al., 2002).

O aumento da ocorrência de florações de cianobactérias tóxicas, associadas a eventos de contaminação da água e morte de humanos e animais, estimulou a Organização Mundial da Saúde (OMS) a publicar protocolos e diretrizes de monitoramento das águas usadas para abastecimento público (CHORUS; BARTRAM, 1999). A maior preocupação são as cianobactérias, potenciais produtoras de cianotoxinas, 
com efeitos hepatotóxicos, neurotóxicos e dermatotóxicos aos indivíduos que ingerirem água ou alimentos contaminados.

Com base nas diretrizes da OMS, a portaria no 518/2004 do Ministério da Saúde (BRASIL, 2004), que estabelece o padrão de potabilidade brasileiro, determina que os mananciais superficiais utilizados para abastecimento público sejam monitorados mensalmente quanto ao número de células de cianobactérias por $\mathrm{mL}$ de água até 10.000 células. $\mathrm{mL}^{-1}$. Acima desse limite, o monitoramento do manancial deve ser semanal. A análise da cianotoxina microcistina passa a ser obrigatória na água tratada da saída do tratamento e nas entradas de clínicas de hemodiálise e indústrias de injetáveis, quando o número de células de cianobactérias no manancial ultrapassar 20.000 células. $\mathrm{mL}^{-1}$.

Considerando que a saúde e o bem-estar humano, bem como o equilíbrio ecológico, não devem ser afetados pela deterioração da qualidade das águas e que o controle da poluição está diretamente relacionado à proteção da saúde e à melhoria da qualidade de vida, foi estabelecida a legislação ambiental resolução 357 do Conselho Nacional de Meio Ambiente (CONAMA), de 2005. Essa resolução, que "dispõe sobre a classificação dos corpos de água e diretrizes ambientais para o seu enquadramento, bem como estabelece as condições e padrões de lançamento de efluentes, e dá outras providências" (CONAMA, 2005), também considera o número de células de cianobactérias como um parâmetro a ser avaliado.

Dessa forma, os laboratórios prestadores de serviço e os laboratórios das empresas responsáveis pelo tratamento da água devem ser capacitados para correta identificação e quantificação desses organismos. Os métodos de contagem utilizados pelos laboratórios são os descritos na bibliografia especializada, sem uma padronização em nível nacional. As metodologias clássicas de identificação e quantificação são baseadas em características morfológicas dos organismos visíveis ao microscópio ótico. Assim, os analistas devem ter habilidade e experiência na identificação dos organismos presentes nas amostras, bem como acesso à literatura específica (chaves de identificação, manuais ilustrados, entre outros) e a cursos para treinamento contínuo (KARLSON et al., 2010). Ainda assim, ensinamentos e orientações de taxonomistas especialistas podem ser necessários.

A participação dos laboratórios em ensaios de proficiência por comparações interlaboratoriais é uma maneira de avaliar seu desempenho na execução de ensaios específicos e verificar a adequação do seu método analítico à finalidade proposta. Esses ensaios representam programas de controle de qualidade amplamente aceitos, tendo como objetivos: 1) monitorar o desempenho contínuo dos laboratórios; 2) identificar problemas em laboratórios e sinalizar a necessidade de ações corretivas; 3 ) identificar diferenças interlaboratoriais e 4) fornecer confiança adicional aos clientes do laboratório (ALBANO; RAYA-RODRIGUEZ, 2009; RMRS, 2010).

Nesse contexto, foi organizado pela Rede Metrológica do Rio Grande do Sul (RMRS) um ensaio de proficiência por comparações interlaboratoriais em cianobactérias inédito em âmbito nacional. Inicialmente, foi realizada uma consulta para verificar a receptividade do programa e o número de potenciais participantes. Em função de sua ampla aceitação, o programa foi realizado em maio de 2010. Os objetivos deste trabalho foram apresentar os resultados e avaliar a situação dos laboratórios brasileiros no que diz respeito à análise de cianobactérias em amostras de água utilizadas para abastecimento público.

\section{Metodologia}

A Secretaria Executiva da RMRS - com o devido apoio do Subcomitê de Microbiologia do Comitê Temático de Meio Ambiente - organizou um programa piloto de ensaio de proficiência em cianobactérias, em maio de 2010. A RMRS é certificada pela ISO 9001 desde 1997 e, em fevereiro de 2004, teve incluído no seu escopo de certificação o processo de "provisão de programas de comparações interlaboratoriais/ensaios de proficiência". É uma das maiores provedoras de ensaios de proficiência da América do Sul, cadastrada no European Proficiency Testing Information System (EPTIS) desde novembro de 2006.

\section{Itens de ensaio}

As amostras foram preparadas a partir de água natural, proveniente de um manancial superficial utilizado para abastecimento público. Após coletada e homogeneizada, a amostra foi fracionada e envazada em frascos de polietileno, com capacidade para 1L. As amostras foram identificadas e acondicionadas em caixas térmicas com gelo, para distribuição aos laboratórios participantes. Cada participante recebeu um frasco contendo amostra preservada com solução de Lugol, preparada segundo APHA; AWWA; WEF, (2005), e um frasco contendo amostra não preservada. Foram avaliadas a identificação e a contagem total de cianobactérias. A análise foi realizada em duplicata e a quantificação, expressa em células. $\mathrm{mL}^{-1}$. De acordo com os métodos normalizados para contagem de cianobactérias, tendo como referência APHA; AWWA; WEF (2005), a identificação dos organismos deve ser realizada na amostra não preservada e a contagem deve utilizar a amostra preservada.

\section{Envio e análise dos resultados}

Após o recebimento das amostras, cada laboratório definiu o momento de iniciar os ensaios. O método utilizado não foi padronizado, ou seja, cada laboratório deveria realizar as análises utilizando o seu método de rotina. Depois de encerradas as análises, cada laboratório enviou seus resultados à RMRS, com código de sigilo.

Para avaliação dos resultados enviados, utilizou-se a estatística robusta. Ao contrário da estatística clássica, a robusta sofre pouca 
influência de valores dispersos (outliers), o que dispensa a utilização de procedimentos para a identificação e remoção desses valores.

O desempenho de cada laboratório participante deste ensaio de proficiência foi avaliado a partir da análise estatística dos resultados enviados, sendo definida a estimativa do valor real (valor designado) por meio de consenso, seguindo-se as diretrizes da norma ISO/DIS 13528 (ISO, 2005). Para avaliação desse desempenho, foi utilizado o escore $z$, seguindo-se as orientações da NBR ISO/IEC Guia 43-1:1999 (ABNT, 2005) e do ILAC-G13:08 (ILAC, 2007). Cada laboratório foi avaliado com relação à média da duplicata de seus resultados, sendo informado, também, o coeficiente de variação (CV) do grupo de laboratórios participantes.

O escore $z$ da média das duplicatas de cada laboratório, calculado utilizando-se como referência o documento ISO (2005), classifica o desempenho dos laboratórios como "satisfatório", "questionável" ou "insatisfatório", conforme o valor do escore z:

Se:

$|z| \leq 2$, resultado satisfatório

$2<|z|<3$, resultado questionável

$|z| \geq 3$,resultado insatisfatório

Os resultados também foram avaliados por meio de análise de variância (ANOVA) para verificar a equivalência entre os métodos de contagem utilizados pelos laboratórios.

\section{Testes de homogeneidade e estabilidade}

Uma análise estatística, com relação à homogeneidade e à estabilidade das amostras preservadas do programa, foi realizada visando verificar se a variabilidade proveniente da eventual falta de homogeneidade ou estabilidade não foi significante perante a variabilidade total dos ensaios. Os testes de homogeneidade e estabilidade foram realizados conforme a norma ISO/DIS 13528 (ISO, 2005).

Para o teste de homogeneidade, foram analisadas dez amostras em duplicata, imediatamente após a preparação e distribuição das amostras aos participantes. Para avaliação da homogeneidade, a referida norma adota um critério para garantir que o desvio padrão entre as amostras contribua com não mais do que 30\% para o desvio padrão do ensaio de proficiência.

Para o teste da estabilidade, foram selecionadas, aleatoriamente, três amostras do teste de homogeneidade que foram analisadas em duplicata, uma semana após a preparação e distribuição das amostras aos participantes (ISO, 2005).

\section{Resultados}

O programa piloto ocorreu por meio de uma rodada de análises. Participaram desse programa 30 laboratórios de todo o país, entre eles, laboratórios de empresas de saneamento, prestadores de serviços e da área da Saúde.
Os resultados do teste de homogeneidade na preparação das amostras são apresentados na Tabela 1 .

Para as amostras serem consideradas homogêneas, o critério de aceitação, segundo a norma ISO/DIS 13528 (ISO, 2005), é que o desvio entre as amostras do teste de homogeneidade $\left(\mathrm{s}_{\mathrm{s}}=311 / \mathrm{em}\right.$ logaritmo: $\left.s_{s}=0,02\right)$ deve ser menor do que 0,3 vezes o desvio do grupo de laboratórios participantes $(0,3 \mathrm{x}$ desvio robusto $=0,3 \mathrm{x}$ $10.705=3.212 /$ em logaritmo: $0,3 \times$ desvio robusto $=0,3 \times 0,23=$ $0,07)$. Portanto, as amostras foram consideradas homogêneas.

A Tabela 2 apresenta os dados do teste de estabilidade na preparação das amostras.

Para as amostras serem consideradas estáveis, o critério de aceitação, segundo a norma ISO/DIS 13528 (ISO, 2005), é que o módulo da diferença $(|x-y|=723$ / em logaritmo: $|x-y|=0,03)$ da média obtida no teste de homogeneidade ( $x=14.123$ / em logaritmo: $x=4,14) e$ da média obtida no teste de estabilidade ( $\mathrm{y}=14.847$ / em logaritmo: $y=4,17$ ) deve ser menor ou igual do que 0,3 vezes o desvio do grupo de laboratórios participantes $(0,3 \times$ desvio robusto $=0,3 \times 10.705$ $=3.212$ / em logaritmo: $0,3 \times$ desvio robusto $=0,3 \times 0,23=0,07$ ). Portanto, as amostras foram consideradas estáveis.

As metodologias utilizadas pelos participantes correspondem a métodos microscópicos para quantificação do fitoplâncton. Dezesseis laboratórios utilizaram o método de Sedgwick-Rafter e 14 utilizaram o método de Utermöhl (RMRS, 2010) (Tabela 1).

Tabela 1 - Resultados do teste de homogeneidade na preparação das amostras

\begin{tabular}{lcccc|}
\multirow{2}{*}{ Amostra } & \multicolumn{2}{c}{$\begin{array}{c}\text { Total de cianobactérias } \\
\left(\text { células. } \mathrm{mL}^{-1} \text { ) }\right.\end{array}$} & \multicolumn{2}{c}{$\begin{array}{c}\text { Total de cianobactérias } \\
\text { (logaritmo) }\end{array}$} \\
\cline { 2 - 5 } & Média & Amplitude & Média & Amplitude \\
\hline 1 & 14.254 & 5.404 & 4,15 & 0,17 \\
\hline 2 & 10.633 & 2.597 & 4,02 & 0,11 \\
\hline 3 & 13.417 & 4.275 & 4,12 & 0,14 \\
\hline 4 & 14.969 & 295 & 4,18 & 0,01 \\
\hline 5 & 13.796 & 3.513 & 4,14 & 0,11 \\
\hline 6 & 14.841 & 2.470 & 4,17 & 0,07 \\
\hline 7 & 16.570 & 2.465 & 4,22 & 0,06 \\
\hline 8 & 13.705 & 1.536 & 4,14 & 0,05 \\
\hline 9 & 15.337 & 3.429 & 4,18 & 0,1 \\
\hline 10 & 13.715 & 479 & 4,14 & 0,02 \\
\hline
\end{tabular}

Fonte: RMRS, 2010

Tabela 2 - Resultados do teste de estabilidade na preparação das amostras

\begin{tabular}{lcccc} 
Amostra & \multicolumn{2}{c}{$\begin{array}{c}\text { Total de cianobactérias } \\
\left(\text { células. } \mathrm{mL}^{-1} \text { ) }\right.\end{array}$} & \multicolumn{2}{c}{$\begin{array}{c}\text { Total de cianobactérias } \\
\text { (logaritmo) }\end{array}$} \\
\cline { 2 - 5 } & Média & Amplitude & Média & Amplitude \\
\hline 7 & 14.503 & 2.323 & 4,16 & 0,06 \\
\hline 8 & 15.046 & 301 & 4,18 & 0,01 \\
9 & 14.993 & 3.571 & 4,17 & 0,1 \\
\hline
\end{tabular}

Fonte: RMRS, 2010 
Tabela 3 - Resultados da rodada piloto para contagem total de cianobactérias

\begin{tabular}{|c|c|c|c|c|c|c|}
\hline Laboratório & $\begin{array}{c}1^{\mathrm{a}} \text { via } \\
\text { (células. } \mathrm{mL}^{-1} \text { ) }\end{array}$ & $\begin{array}{c}2^{\mathrm{a}} \text { via } \\
\text { (células. } \mathrm{mL}^{-1} \text { ) }\end{array}$ & $\begin{array}{c}1^{\mathrm{a}} \text { via } \\
\text { (logaritmo) }\end{array}$ & $\begin{array}{c}2^{\mathrm{a}} \text { via } \\
\text { (logaritmo) }\end{array}$ & $\begin{array}{c}\text { z escore } \\
\text { (logaritmo) }\end{array}$ & $\begin{array}{l}\text { Método de } \\
\text { contagem }\end{array}$ \\
\hline 1 & 7.431 & 7.673 & 3,87 & 3,88 & $-1,65$ & Utermöhl \\
\hline 2 & 17.364 & 19.584 & 4,24 & 4,29 & 0,01 & Sedgwick-Rafter \\
\hline 3 & 35.531 & 38.819 & 4,55 & 4,59 & 1,31 & Utermöhl \\
\hline 4 * & 2.633 & 2.728 & 3,42 & 3,44 & $-3,58$ & Utermöhl \\
\hline 5 & 37.603 & 43.967 & 4,58 & 4,64 & 1,48 & Utermöhl \\
\hline 6 & 27.678 & 19.012 & 4,44 & 4,28 & 0,45 & Sedgwick-Rafter \\
\hline 7 & 28.785 & 30.785 & 4,46 & 4,49 & 0,9 & Utermöhl \\
\hline 8 & 8.599 & 31.597 & 3,93 & 4,5 & 0,17 & Utermöhl \\
\hline 9 & 25.932 & 24.883 & 4,41 & 4,4 & 0,6 & Utermöhl \\
\hline 10 * & 1.484 & 1.370 & 3,17 & 3,14 & $-4,75$ & Sedgwick-Rafter \\
\hline 11 * & 2.430 & 2.315 & 3,39 & 3,36 & $-3,8$ & Sedgwick-Rafter \\
\hline 12 * & 1.052 .790 & 957.315 & 6,02 & 5,98 & 7,43 & Sedgwick-Rafter \\
\hline 13 & 21.668 & 22.233 & 4,34 & 4,35 & 0,33 & Sedgwick-Rafter \\
\hline 14 & 17.091 & 13.917 & 4,23 & 4,14 & $-0,32$ & Utermöhl \\
\hline 15 & 31.689 & 30.653 & 4,5 & 4,49 & 0,98 & Utermöhl \\
\hline 16 & 30.811 & 30.151 & 4,49 & 4,48 & 0,94 & Sedgwick-Rafter \\
\hline 17 & 19.371 & 19.453 & 4,29 & 4,29 & 0,1 & Sedgwick-Rafter \\
\hline 18 & 21.419 & 22.664 & 4,33 & 4,36 & 0,34 & Sedgwick-Rafter \\
\hline 19 & 17.359 & 20.513 & 4,24 & 4,31 & 0,06 & Sedgwick-Rafter \\
\hline 20 & 22.324 & 19.336 & 4,35 & 4,29 & 0,23 & Sedgwick-Rafter \\
\hline 21 & 5.693 & 17.043 & 3,76 & 4,23 & $-0,89$ & Utermöhl \\
\hline 22 & 24.077 & 26.695 & 4,38 & 4,43 & 0,6 & Sedgwick-Rafter \\
\hline 23 ** & 6.025 & 4.425 & 3,78 & 3,65 & $-2,34$ & Sedgwick-Rafter \\
\hline 24 * & 3.738 & 3.052 & 3,57 & 3,48 & $-3,14$ & Sedgwick-Rafter \\
\hline 25 & 10.595 & 9.418 & 4,03 & 3,97 & $-1,13$ & Utermöhl \\
\hline 26 & 13.500 & 11.010 & 4,13 & 4,04 & $-0,75$ & Sedgwick-Rafter \\
\hline 27 & 17.128 & 14.769 & 4,23 & 4,17 & $-0,26$ & Utermöhl \\
\hline 28 & 15.951 & 16.455 & 4,2 & 4,22 & $-0,23$ & Sedgwick-Rafter \\
\hline 29 & 23.978 & 25.229 & 4,38 & 4,4 & 0,54 & Utermöhl \\
\hline 30 & 27.388 & 19.251 & 4,44 & 4,28 & 0,44 & Utermöhl \\
\hline
\end{tabular}

Fonte: RMRS, 2010

*Laboratório com desempenho insatisfatório; **Laboratório com desempenho questionável

A Tabela 3 apresenta os resultados das contagens, em duplicata, de cada participante e o escore z de cada um. Os dados também foram analisados após transformação para logaritmo base 10, pois como são dados de contagem, são mais bem avaliados após transformação, já que estabiliza a variação entre os resultados (ISO, 2006b). $\mathrm{Na}$ Tabela 2, são apresentados os parâmetros estatísticos.

Dos 30 laboratórios participantes, 24 apresentaram desempenho satisfatório, 1 apresentou desempenho questionável e 5 apresentaram desempenho insatisfatório.

A ANOVA mostrou equivalência entre os métodos de contagem de Sedgwick-Rafter e de Utermöhl ( $F=0,027 ; p=0,97$ ) (Tabela 4).

Em relação à análise qualitativa, verificou-se maior divergência entre os participantes (Tabela 5), sendo que somente um laboratório não identificou o organismo predominante (Planktothrix sp.).
Muitos laboratórios informaram que não puderam identificar os gêneros da família Nostocaceae devido à ausência de estruturas (acinetos e heterocitos) que permitem a sua diferenciação. Assim, na Tabela 5, Nostocaceae inclui os gêneros Aphanizomenon, Cylindrospermopsis, Cuspidothrix e Raphidiopsis, identificados pelos participantes.

\section{Discussão}

Comparações interlaboratoriais como esta, realizada por meio do programa piloto de ensaios de proficiência em cianobactérias, não são comuns para fitoplâncton, nem para o grupo das cianobactérias. A quantificação desses organismos é uma exigência da portaria $n^{\circ}$ 518/2004 do Ministério da Saúde brasileiro (BRASIL, 2004) e da 
Tabela 4 - Avaliação estatística dos resultados enviados pelos laboratórios participantes

\begin{tabular}{|c|c|c|}
\hline & 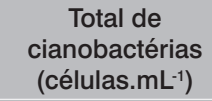 & $\begin{array}{c}\text { Total de } \\
\text { cianobactérias } \\
\text { (logaritmo) }\end{array}$ \\
\hline & \multicolumn{2}{|c|}{ Total de laboratórios participantes ( 30} \\
\hline Média robusta & 19.099 & 4,26 \\
\hline Desvio robusto & 10.705 & 0,23 \\
\hline № total de laboratórios & 30 & 30 \\
\hline \multirow[t]{2}{*}{ Coeficiente de variação (\%) } & 56 & 5,48 \\
\hline & \multicolumn{2}{|c|}{$\begin{array}{l}\text { Laboratórios que utilizaram } \\
\text { método de Utermöhl }\end{array}$} \\
\hline Média robusta & 21.101 & 4,29 \\
\hline Desvio robusto & 12.806 & 0,25 \\
\hline № total de laboratórios & 14 & 14 \\
\hline \multirow[t]{2}{*}{ Coeficiente de variação (\%) } & 61 & 5,74 \\
\hline & \multicolumn{2}{|c|}{$\begin{array}{l}\text { Laboratórios que utilizaram } \\
\text { método de Sedgwick-Rafter }\end{array}$} \\
\hline Média robusta & 18.159 & 4,26 \\
\hline Desvio robusto & 8.833 & 0,18 \\
\hline № total de laboratórios & 16 & 16 \\
\hline Coeficiente de variação (\%) & 49 & 4,18 \\
\hline
\end{tabular}

Fonte: RMRS, 2010

Resolução CONAMA 357/2005 (CONAMA, 2005); por isso, há importância em avaliar o desempenho dos laboratórios que realizam esse ensaio no país.

Estudos de comparação interlaboratorial já foram realizados por Gohde et al. (2007) e Vuorio, Lepistö e Holopainen (2007), porém, com formatos diferenciados. O primeiro estudo fez a comparação entre métodos clássicos e métodos moleculares para uma única espécie de dinoflagelado marinho. O segundo avaliou organismos específicos, informados no início do estudo, e empregou método e orientações de contagem iguais para todos os participantes. A proposta do programa promovido pela RMRS foi diferente, seguindo-se a norma ISO/DIS 13528 (ISO, 2005); nessa proposta, os laboratórios recebiam a mesma amostra, mas o preparo, método e critérios de contagem seguiam a rotina de análise de cada participante.

Os testes de homogeneidade e estabilidade, indicando que as amostras foram homogêneas e estáveis nas condições consideradas, mostram que, mesmo os organismos distribuindo-se de forma agregada e formando suspensão com variabilidade inerente (ISO, 2006a), é possível reproduzir os resultados em amostras fracionadas. Com a agitação da amostra, que deve ocorrer de forma suave para não romper os arranjos coloniais, atinge-se a homogeneidade. Em relação à estabilidade, confirma-se a eficácia da solução de Lugol quando as amostras são refrigeradas e mantidas no escuro (EDLER; ELBRÄCHTER, 2010).

Os resultados quantitativos, apresentados pela maioria dos laboratórios participantes do programa, indicam que os métodos de contagem utilizados são adequados para a quantificação das
Tabela 5 - Gêneros de cianobactérias identificados pelos laboratórios participantes

\begin{tabular}{|c|c|}
\hline Gêneros identificados & $\begin{array}{l}\mathrm{N}^{\circ} \text { de laboratórios que } \\
\text { identificaram o gênero }\end{array}$ \\
\hline Anabaena & 2 \\
\hline Aphanocapsa & 6 \\
\hline Calothrix & 1 \\
\hline Chroococcus & 4 \\
\hline Coelomorum & 1 \\
\hline Coelosphaerium & 1 \\
\hline Geitlerinema & 11 \\
\hline Jaaginema & 1 \\
\hline Limnothrix & 5 \\
\hline Merismopedia & 20 \\
\hline Microcystis & 12 \\
\hline Myxobaktron & 1 \\
\hline Oscillatoria & 5 \\
\hline Phormidium & 3 \\
\hline Planktolyngbya & 5 \\
\hline Planktothix & 29 \\
\hline Pseudanabaena & 13 \\
\hline Rhabdoderma & 1 \\
\hline Romeria & 2 \\
\hline Snowella & 1 \\
\hline Sphaerocavum & 2 \\
\hline Spirulina & 1 \\
\hline Synechococcus & 3 \\
\hline Synechocystis & 2 \\
\hline Tychonema & 1 \\
\hline Wolskyella & 1 \\
\hline Nostocaceae* & 26 \\
\hline Não identificado & 2 \\
\hline
\end{tabular}

cianobactérias. O método de Utermöhl é recomendado para a quantificação de cianobactérias (CHORUS; BARTRAM, 1999; CETESB, 2005; CEN, 2006; EPA, 2007) e caracteriza-se por ser um método que demanda mais tempo para a realização da análise (em função do longo tempo para sedimentação das câmaras de contagem) e um custo com equipamento mais elevado (necessidade de microscópio específico, isto é, microscópio invertido) (EDLER; ELBRÄCHTER, 2010). Os resultados mostram que o método de Sedgwick-Rafter (BRANCO, 1986; ASTM, 2004; APHA; AWWA; WEF, 2005; CETESB, 2005; LEGRESLEY; MCDERMOTT, 2010) também pode ser utilizado para a contagem desses organismos. Esse método permite maior rapidez na obtenção do resultado e não requer equipamento específico. A utilização de microscópio ótico comum o torna um método de custo mais baixo (EDLER; ELBRÄCHTER, 2010; LEGRESLEY; MC DERMOTT, 2010). Entretanto, cabe ressaltar que o resultado pode 
ser sub ou superestimado, em função dos fatores de correção que devem ser utilizados para obtenção do resultado final (GOHDE et al., 2007); por isso, deve-se ter muito cuidado, para um correto preparo da amostra (concentração ou diluição, quando for o caso). Dentre os laboratórios que apresentaram desempenho insatisfatório, 80\% utilizaram esse método de contagem.

Comparando-se com programas de proficiência envolvendo ensaios microbiológicos da RMRS, o CV obtido neste programa piloto é mais baixo (56\%). Quanto maior o CV, maior o número de laboratórios na categoria de desempenho aceitável, pois a faixa de aceitabilidade fica aumentada (RMRS, 2010). Nos ensaios microbiológicos, são comuns CVs maiores do que 100\%. Dessa forma, todos os laboratórios devem avaliar criticamente seu procedimento de análise a fim de reduzir o $\mathrm{CV}$ em rodadas futuras, aumentando a precisão dos resultados (BRUCE; MINKKINEN; RIEKKOLA, 1998; ALBANO; RAYA-RODRIGUEZ, 2009; INMETRO, 2010).

Embora não seja o método mais recomendado pela literatura especializada para a quantificação de cianobactérias, os resultados mostraram que o método de Sedgwick-Rafter é adequado a essa finalidade, fornecendo resultados satisfatórios e semelhantes aos resultados obtidos pelo método de Utermöhl. Além disso, foi o método com menor variabilidade, isto é, apresentou um CV de 49\%, menor do que o método de Utermöhl $(\mathrm{CV}=61 \%)$ e do que os dois métodos em conjunto ( $C V=56 \%$ ) (Tabela 1$)$. Isso indica uma menor faixa de variação entre os resultados dos laboratórios que realizaram a análise por meio desse método.

Em relação à análise qualitativa (Tabela 5), poucos gêneros foram encontrados pela maioria dos laboratórios. Em contrapartida, muitos gêneros foram encontrados por somente um participante. Dentre os gêneros apresentados, muitos podem ter identificação dificultada. Calothrix, Myxobaktron, Spirulina, Synechocystis e Synechococcus têm ocorrência rara ou poucas espécies já foram mencionadas no Brasil (WERNER, 2002; BICUDO; MENEZES, 2006). Os três primeiros gêneros ainda podem ser confundidos com o gênero Cylindrospermopsis, que apresenta grande variação morfológica (KOMÁREK; KLING, 1991).

Os gêneros Jaaginema e Romeria são morfologicamente semelhantes à Geitlerinema, assim como Tychonema pode ser confundido com Oscillatoria, Sphaerocavum com Microcystis e Rhabdoderma com Aphanocapsa e Aphanotece. Ainda há organismos com filamentos finos ou mesmo células isoladas, como Limnothrix e Planktolyngbya ou Synechococcus, respectivamente, que, quando observados em pequenos aumentos, podem ser confundidos com bactérias heterotróficas. O uso de microscópio com epifluorescência auxilia nessa diferenciação, pois a clorofila presente nos organismos autotróficos apresenta autofluorescência (GREGOR; MARŠÁLEK, 2004; MACEDO; MARINO, 2007; KARLSON et al., 2010). Organismos pertencentes ao picoplâncton, como algumas espécies dos gêneros Snowella, Coelomorum e Coelosphaerium (KOMÁREK; ANAGNOSTIDIS, 1998), que apresentam arranjo colonial semelhante entre si, podem ter identificação imprecisa. Isso pode ocorrer devido aos métodos utilizados pelos participantes que não são recomendados para identificação e quantificação de organismos dessa categoria (VUORIO; LEPISTÖ; HOLOPAINEN, 2007; LEGRESLEY; MCDERMOTT, 2010).

As divergências na identificação, o registro de organismos não identificados e a dificuldade para a diferenciação dos gêneros da família Nostocaceae podem indicar falta de conhecimento ou de treinamento adequado dos analistas. Enquanto a legislação exigir a quantificação do grupo das cianobactérias, os resultados apresentados pelos laboratórios são coerentes. Porém, uma identificação correta dos gêneros presentes também é importante. De acordo com os gêneros encontrados, é possível avaliar a potencialidade da produção de cianotoxinas e realizar a análise apropriada, se necessário (CHORUS; BARTRAM, 1999; SANT'ANNA et al., 2006; CYBIS et al., 2006).

\section{Conclusões}

O programa piloto de ensaios de proficiência em cianobactérias pode ser considerado uma primeira avaliação de desempenho de alguns laboratórios brasileiros responsáveis pela análise de cianobactérias em mananciais superficiais. Assim como os demais programas dessa natureza, seus objetivos foram atingidos (ALBANO; RAYA-RODRIGUEZ, 2009; RMRS, 2010). Os métodos analíticos utilizados pelos laboratórios, a saber, método de Sedgwick-Rafter e método de Utermöhl, mostraram-se adequados e equivalentes para a quantificação de cianobactérias, não havendo necessidade de uma padronização de método de ensaio. De maneira geral, os laboratórios estão desempenhando bem a tarefa de quantificar esses organismos. Com base nos resultados obtidos, que servem como uma ferramenta de melhoria contínua, os laboratórios devem avaliar a identificação das cianobactérias e, se pertinente, propor internamente ações corretivas, preventivas e/ou oportunidades de melhoria. Os laboratórios com desempenho questionável e insatisfatório na análise quantitativa também devem realizar análise crítica em relação ao seu método de contagem, sempre buscando melhoria contínua na confiabilidade analítica.

\section{Agradecimentos}

Agradecemos à Rede Metrológica do Rio Grande do Sul, pela provisão do programa piloto de ensaios de proficiência em cianobactérias e autorização para divulgação dos dados do seu programa, e ao Laboratório Central de Águas da Companhia Riograndense de Saneamento, em especial, Andréa dos Anjos, Aurea Giordani e Juliana Frizzo, pelo fornecimento e preparação das amostras, utilização de suas instalações e apoio na realização das análises. 


\section{Referências}

ALBANO, F.M.; RAYA-RODRIGUEZ, M.T. Validação e garantia da qualidade de ensaios laboratoriais: guia prático. Porto Alegre: Rede Metrológica RS, 2009.

AMERICAN SOCIETY FOR TESTING AND MATERIALS (ASTM). ASTM D4148-82: standard test method for analysis of phytoplankton in surface water by the sedgwick-rafter method. West Conshohocken, PA, USA: ASTM, 2004.

APHA; AWWA; WEF. Standard methods for the examination of water and wastewater. 21 ed. Washington, D.C., 2005.

ASSOCIAÇÃO BRASILEIRA DE NORMAS TÉCNICAS (ABNT). NBR ISO/IEC Guia 43-1:1999: ensaios de proficiência por comparações interlaboratoriais - Parte 1: desenvolvimento e operação de programas de ensaios de proficiência. Rio de Janeiro: ABNT, 2005.

BICUDO, C.E.M.; MENEZES, M. (Orgs.). Gêneros de algas de águas continentais do Brasil: chave para identificação e descrições. 2 ed. São Carlos: RiMa, 2006.

BRANCO, S.M. Hidrobiologia aplicada à Engenharia Sanitária. 3 ed.. São Paulo: CETESB/ASCETESB, 1986.

BRASIL. Ministério da Saúde. Portaria n 518, de 25 de março de 2004: estabelece os procedimentos e responsabilidades relativos ao controle e vigilância da qualidade da água para consumo humano e seu padrão de potabilidade, e dá outras providências. Brasília: Diário Oficial da União, n. 59, p. 266-270, 2004.

BRUCE, P.; MINKKINEN, P.; RIEKKOLA, M.L. Practical method validation: validation sufficient for an analysis method. Mikrochimica Acta. v. 128, n. 1-2, p. 93-106, 1998

CHORUS, I.; BARTRAM, J. Toxic cyanobacteria in water: a guide to their public health consequences, monitoring and management. London: Spon E \& EN, 1999.

COMPANHIA DE TECNOLOGIA DE SANEAMENTO AMBIENTAL (CETESB). Norma Técnica L5.303: fitoplâncton de água doce - métodos qualitativo e quantitativo (método de ensaio). São Paulo, 2005.

CONSELHO NACIONAL DO MEIO AMBIENTE (CONAMA). Resolução CONAMA $n^{\circ}$ 357. Dispõe sobre a classificação dos corpos de água e diretrizes ambientais para o seu enquadramento, bem como estabelece as condições e padrões de lançamento de efluentes, e dá outras providências. Brasília: Diário Oficial da União, n. 53, p. 58-63, 2005.

CYBIS, L.F. et al. Manual para estudo de cianobactérias planctônicas em mananciais de abastecimento público: caso da represa Lomba do Sabão e Lago Guaíba, Porto Alegre, Rio Grande do Sul. Rio de Janeiro: ABES, 2006.

EDLER, L.; ELBRÄCHTER, M. The Utermöhl method for quantitative phytoplankton analysis. In: KARLSON, B.; CUSACK, C.; BRESNAN, E. (Eds.) Microscopic and molecular methods for quantitative phytoplankton analysis. UNESCO. (IOC Manuals and Guides, n 55.) (IOC/2010/MG/55), Paris: United Nations Educational, Scientific and Cultural Organization, 2010, p. 13-20.
ENVIRONMENTAL PROTECTION AGENCY (EPA). LG401: Standard Operating Procedure for Phytoplankton Analysis. Revision 4. Washington, DC, 2007.

EUROPEAN COMMITTEE FOR STANDARDIZATION (CEN). EN 15204: Water Quality - Guidance standard on the enumeration of phytoplankton using inverted microscopy (Utermöhl technique). Brussels: CEN, 2006.

GOHDE, A. et al. Intercalibration of classical and molecular techniques for identification of Alexandrium fundyense (Diniphyceae) and estimation of cell densities. Harmful Algae, v. 6, p. 56-72, 2007.

GREGOR, J. et al. In situ quantification of phytoplankton in reservoirs using a submersible spectrofluorometer. Hydrobiologia, v. 548, n. 1, p. 141-151, 2005.

GREGOR, J.; MARŠÁLEK, B. Freshwater phytoplankton quantification by chlorophyll a: a comparative study of in vitro, in vivo and in situ methods. Water Research, v. 38, n. 3, p. 517-522, 2004.

INSTITUTO NACIONAL DE METROLOGIA, NORMALIZAÇÃO E QUALIDADE INDUSTRIAL (INMETRO). 2010. Orientação sobre Validação de Métodos Analíticos. DOQ-CGCRE-008: Revisão 03. Disponíve em <http://www.inmetro.gov.br/Sidoq/Arquivos/CGCRE/DOQ/DOQCGCRE-8_03.pdf > . Acesso em: 12 jul. 2010.

INTERNATIONAL LABORATORY ACCREDITATION COOPERATION (ILAC). ILAC-G13:08-Guidelines for the Requirements for the Competence of Providers of Proficiency Testing Schemes. ILAC: Austrália, 2007.

INTERNATIONAL ORGANIZATION FOR STANDARDIZATION (ISO). ISO 19458. Water quality - Sampling for microbiological analysis. Geneva: ISO, 2006a.

ISO/TS 19036. Microbiology of food and animal feeding stuffS Guidelines of the estimation of measurement uncertainty for quantitative determinations. Geneva: ISO, 2006b.

ISO 13528. Statistical methods for use in proficiency testing by interlaboratory comparisons. Geneva: ISO, 2005.

KARLSON, B. et al. Introduction to methods for quantitative phytoplankton analysis. In: KARLSON, B.; CUSACK, C.; BRESNAN, E. (Eds.) Microscopic and molecular methods for quantitative phytoplankton analysis. UNESCO (IOC Manuals and Guides, n 55.) (IOC/2010/MG/55), Paris: United Nations Educational, Scientific and Cultural Organization, 2010, p. 5-12.

KOMÁREK, J.; ANAGNOSTIDIS, K. Cyanoprokaryota. 1. Teil: Chroococcales. In: PASCHER, A. (Ed.) Süßwasserflora von Mitteleuropa 19/1. Stuttgart: Gustav Fischer, 1998, p. 192-224.

KOMÁREK, J.; KLING, H. Variation in six planktonic cyanophyte genera in Lake Victoria (East Africa). Algological Studies, v. 61, p. 21-45, 1991.

LEBOULANGER, C. etal. Application of a submersible spectrofluorometer for rapid monitoring of freshwater cyanobacterial blooms: a case study. Aquatic Microbial Ecology, v. 30, p. 83-89, 2002. 
LEGRESLEY, M.; MCDERMOTT, G. Counting chamber methods for quantitative phytoplankton analysis - haemocytometer, PalmerMaloney cell and Sedgewick-Rafter cell. In: KARLSON, B.; CUSACK, C.; BRESNAN, E. (Eds.) Microscopic and molecular methods for quantitative phytoplankton analysis. UNESCO (IOC Manuals and Guides, $n^{\circ}$ 55.) (IOC/2010/MG/55), Paris: United Nations Educational, Scientific and Cultural Organization, 2010, p. 25-30.

MACEDO, A.; MARINO, L. O microscópio de epifluorescência como auxiliar na identificação de cianobactérias. In: CONGRESSO BRASILEIRO DE ENGENHARIA SANITÁRIA E AMBIENTAL, 24, Anais... Belo Horizonte: ABES, I-176, 2007.

REDE DE METROLOGIA E ENSAIOS DO RIO GRANDE DO SUL (RMRS). Programa Piloto de Ensaios de Proficiência em Cianobactérias: relatório de 2010. Porto Alegre: RMRS, 2010.
SANT'ANNA, C.L. et al. Manual ilustrado para identificação e contagem de cianobactérias planctônicas de águas continentais brasileiras. Rio de Janeiro: Interciência; São Paulo: Sociedade Brasileira de Ficologia, 2006.

VUORIO, K.; LEPISTÖ, L.; HOLOPAINEN, A.L. Intercalibrations of freshwater phytoplankton analyses. Boreal Environment Research, v. 12, n. 5, p. 561-570, 2007.

WERNER, V.R. Cyanophyceae/Cyanobacteria no sistema de lagoas e lagunas da Planície Costeira do Estado do Rio Grande do Sul, Brasil. Tese (Doutorado em Ciências Biológicas), Universidade Estadual Paulista "Júlio de Mesquita Filho", Rio Claro, SP, 2002. 